\title{
Perfil neuropsicológico de pacientes con síndrome de Down
}

Neuropsychological profile of patients with Down syndrome

Ángel F. Villalva-Sánchez ${ }^{a}$, Julio Sandoval-Molina ${ }^{b}$, Luis A. Hernández-Hernández ${ }^{c}$, Ángel O.

Pérez Rodríguez ${ }^{d}$

\begin{abstract}
:
Phenotypic clinical characteristics described in patients with Down Syndrome (DS), however, it is not possible to delve into the cerebral characteristics. Some macro-structural brain alterations have been described, such as the reduction of the frontal lobe cortex, in the anterior angulated gyrus, anterior and posterior parahyppocampal gyrus, hippocampus, amygdala, thalamus, and increase in the third ventricle. On the other hand, in the cognitive area intellectual disability has been related as a generalized alteration in almost all cases, nevertheless, specific deficits have been found in cognitive functions as in expressive and comprehensive language, executive functions, attention, memory, regulation emotional and social cognition. Knowing in detail a neuropsychological profile of patients with DS will give us greater possibility of developing intervention plans that adjust to their needs.
\end{abstract}

Keywords:

Neuropsychology, social cognition, Down syndrome, trisomy 21

\section{Resumen:}

Existe una serie de características clínicas fenotípicas descritas en pacientes con Síndrome de Down (SD), sin embargo, no se ahondado en las características cerebrales. Se han descrito algunas alteraciones cerebrales macroestructurales como la reducción de corteza del lóbulo frontal, en la circunvolución angulada anterior, giro parahipocampal anterior y posterior, hipocampo, amígdala, tálamo y el incremento del tercer ventrículo. Por otro lado, en el área cognitiva se ha relacionado la discapacidad intelectual como una alteración generalizada en casi todos los casos, no obstante, se han encontrado déficits específicos en funciones cognitivas como en lenguaje expresivo y comprensivo, funciones ejecutivas, atención, memoria, regulación emocional y cognición social. Conocer a detalle un perfil neuropsicológico de pacientes con SD nos dará mayor posibilidad de desarrollar planes de intervención que se ajusten a sus necesidades.

\section{Palabras Clave:}

Neuropsicología, cognición social, Síndrome Down, trisomía 21

\section{Introducción}

El Síndrome de Down (SD) fue descrito por primera vez por John Langdon Down en 1866, actualmente también es conocido como trisomía 21 o trastorno cromosómico y es la causa genética más frecuente de discapacidad intelectual. De acuerdo con la Asociación de Psiquiatría Americana (APA, 2014) la discapacidad intelectual es un trastorno que comienza durante el periodo del desarrollo y que incluye limitaciones en el funcionamiento intelectual como también del comportamiento adaptativo en los dominios conceptual, social y práctico. La Organización
Mundial de la Salud (OMS, 2014) menciona que por cada 1100 nacimientos uno presenta SD, algunas instituciones como la Secretaría de Salud de México (SS, 2014) hace referencia a una prevalencia de 1 por cada 650 recién nacidos, en la literatura los niños con síndrome de Down han sido descritos como felices, cariñosos y sociables (Gibbs y Thorpe, 1983).

El Instituto Nacional de Desarrollo Humano Sano del Niño (NICHD, por sus siglas en inglés) (2018) explica las tres variaciones genéticas en el SD. Trisomía 21: se estima que 9 de cada 10 niños con SD está causado por trisomía 21, esto se desarrolla en la fertilización cuando 3 (en vez

\footnotetext{
a Universidad Nacional Autónoma de México, FES Iztacala. Email: angel.villalva@iztacala.unam.mx

${ }^{\mathrm{b}}$ Instituto de Posgrado en Psicoterapia Cognitivo Conductual, IPPCC. Email: julio85molina@gmail.com

c Servicios Educativos Integrados al Estado de México, CAM No. 46. Email: luiscp_crn@yahoo.com.mx

${ }^{\mathrm{d}}$ Pasante de Psicología, Escuela Superior Actopan, UAEH. Email: psicangpr96@ gmail.com
} 
de dos) cromosomas 21 son presentes, generalmente es el óvulo el portador del cromosoma defectuoso, dando finalmente como resultado que todas las células subsecuentes cuenten con tres cromosomas 21 (Stratford, 1998). Trisomía 21 mosaico: es muy similar a la trisomía 21 , la diferencia radica que el cromosoma 21 extra, se extrapola con algunas células, este tipo causa el crecimiento de células anormales de manera aleatoria junto con células normales. Trisomía 21 por translocación: una parte del cromosoma 21 llega a ser translocado a otro cromosoma (regularmente cromosoma 13, 14 ó 15) antes de 0 en la concepción. El portador (cromosoma translocado) tendrá 45 cromosomas en lugar de 46, pero llevando material genético de 46 cromosomas, esto se explica porque el material genético del cromosoma 21 está situado en otro cromosoma.

\section{Hallazgos neuropsicológicos en trisomía 21}

Existe una serie de características clínicas fenotípicas descritas en pacientes con SD como braquicefalia, fisuras palpebrales oblicuas ascendentes, epicanto, manchas de Brushfield, orejas displásicas, puente nasal deprimido, paladar estrecho, cuello corto, piel de nuca redundante, hiperflexibilidad, manos anchas, braquidactilia y pliegue palmar transverso (BMHI, 2014). En específico, en el desarrollo del sistema nervioso central en personas con SD se han descrito diversas anomalías que afectan estructuras neuroanatómicas, p. ej., existe una disminución de neuronas debido a los genes del cromosoma 21 (como el DYIRK1A) que se extienden a varias áreas de la corteza cerebral, al verse una disminución de neuronas en áreas corticales afecta al lóbulo frontal (Pueschel, 1994), el equipo de Raz (1996) halló evidencia de morfometría por resonancia magnética en sujetos con SD, donde destacan las áreas anatómicas como una disminución notable de la corteza prefrontal dorsolateral, la circunvolución cingulada anterior, disminución de la sustancia blanca parietal. De acuerdo a Altafaj et al. (2001), la corteza auditiva está especialmente afectada en la trisomía 21, una marcada disminución de sinapsis en esta región perjudica en los circuitos neuronales del lenguaje, el diámetro anteroposterior disminuye significativamente, debido a la reducción de los lóbulos frontales y al aplanamiento de los occipitales, al verse comprometidas las áreas prefrontales afecta la funcionalidad de las funciones ejecutivas, indispensables para regular el comportamiento humano y la adaptación al contexto, asimismo, se manifiesta una reducción volumétrica de las áreas temporales superiores, comprometiendo las áreas del lenguaje (Snell, 2001). Otro estudio donde se utilizó resonancia magnética de alta resolución donde analizaron los volúmenes de la amígdala y del hipocampo, encontraron una disminución en el hipocampo, pero no en la amígdala (Pinter et al., 2001), un año después Krasuski y Alexander (2002) encontraron que estructuras del lóbulo temporal medial como la amígdala, el hipocampo y el giro parahipocampal anterior y posterior reducen significativamente con la edad en personas con trisomía 21.

Por otro lado, en análisis del desempeño cognitivo Lanfranchi, Cornoldi y Vianello (2004) evaluaron niños con SD en tareas de memoria de trabajo verbal y visuoespacial, encontrando que las tareas que requieren un control bajo, los niños mostraron un deterioro de las tareas de memoria de trabajo verbal pero no visuoespacial, a medida que incrementaba el trabajo, tenía un deterioro en ambas tareas. Schimmel, Hammerman y Bromiker (2006) hallaron diferencias significativas anatómicas en el tercer ventrículo en neonatos con trisomía 21 , en comparación con niños sin signos clínicos de trisomía 21, siendo el tercer ventrículo una estructura funcional que contribuye al desarrollo cognitivo, asimismo, se han encontrado procesos neuropsicológicos deficientes en trisomía 21 como la dificultad del procesamiento de información, atención sostenida disminuida, problemas en el almacenamiento y evocación de memoria a corto plazo, problemas en la memoria declarativa semántica, problemas en la memoria auditiva (Morales y Lopez, 2006).

En estudios como los de Tervo-Maatta (2006) donde evaluaron a niños con trisomía $21(n=129)$ hallaron que las mujeres poseen mejores habilidades cognitivas en comparación a los hombres. Por otro lado durante el neurodesarrollo en la estructura de las neuronas existen anomalías subcelulares y una alteración en la sinaptogénesis del sistema interneuronal (Godoy y Campos, 2014), también se ha encontrado dificultad en el procesamiento e identificación de emociones negativas en niños con trisomía 21 y que se manifiesta en el comportamiento adaptativo y las relaciones interpersonales (Goldman et al, 2016), un año después (Grego del Cole et al, 2017) identificaron las principales dificultades en el perfil cognitivo en SD, donde se encuentran limitaciones en la comunicación, en el procesamiento verbal, memoria a corto plazo, lenguaje expresivo, la gramática y pronunciación, por lo tanto de acuerdo con su estudio proponían los dominios del más fuerte al más débil quedando así: socialización, habilidades de la vida diaria y comunicación, además cabe mencionar que los mismos autores proponen que estas variables neuropsicológicas y psicológicas estarán influencias por el nivel educativo y económico de los padres: 
Del mismo modo, Perry, Pakkenberg y Vann (2018) hallaron en cuatro estudios posmortem de adultos mujeres con trisomía 21 una reducción volumétrica notable de hasta el $70 \%$ en el tálamo, esto involucra una reducción de células gliales, las cuales contribuyen para el proceso neuropsicológico de la memoria espacial y episódica, esto explicaría el estudio de Wu y Morris (2013) donde encontraron un aumento concomitante en el número de adultos con trisomía 21 afectados con demencia, siendo la más común la enfermedad neurodegenerativa del Azheimer. En la tabla 1 se encuentra un resumen de la descripción de niños con trisomía 21.

Tabla 1. Resumen de las características físicas, neuroanatómicas, neuropsicológicas y conductuales de personas con trisomía 21

\begin{tabular}{|c|}
\hline Características físicas \\
\hline $\begin{array}{l}\text { - } \text { Braquicefalia } \\
\text { - } \quad \text { Fisuras palpables oblicuas ascendentes } \\
\text { - } \quad \text { Mancanto } \\
\text { - } \text { Orejas displásicas } \\
\text { - Puente nasal deprimido } \\
\text { - } \quad \text { Paladar estrecho } \\
\text { - } \quad \text { Maello corto } \\
\text { - } \quad \text { Braquidactilia }\end{array}$ \\
\hline Características neuroanatómicas \\
\hline $\begin{array}{ll}\text { - } & \text { Reducción de Corteza cerebral } \\
\text { - } & \text { Lóbulos frontales } \\
\text { - } & \text { Corteza prefrontal dorsolateral } \\
\text { - } & \text { Reducción de la Circunvolución angulada } \\
\text { - } & \text { anterior } \\
\text { - } & \text { Reducción de la Corteza auditiva } \\
\text { - } & \text { Menor Diámetro anterior posterior } \\
\text { - } & \text { Reducción volumétrica en áreas } \\
\text { - } & \text { Remporales superiores } \\
\text { - } & \text { Reducción del hipocampo } \\
\text { - } & \text { Reducción de la amígdala } \\
\text { - } & \text { Reducción del gulo temporal medial } \\
& \text { anterior y posterior } \\
\text { - } & \text { Incremento del tercer ventrículo } \\
\text { - } & \text { Alteración en la sinaptogenesis } \\
\text { - } & \text { Disminución del tálamo y células gliales }\end{array}$ \\
\hline Características neuropsicológicas \\
\hline $\begin{array}{l}\text { - Limitaciones en el Lenguaje: expresivo y } \\
\text { comprensivo } \\
\text { - Limitaciones en las funciones ejecutivas } \\
\text { como la regulación del comportamiento, } \\
\text { regulación de la conducta emocional } \\
\text { - Dificultades en la atención sostenida } \\
\text { - Dificultades en el procesamiento de la } \\
\text { información } \\
\text { - Problemas en el almacenamiento y } \\
\text { evocación de memoria a corto plazo }\end{array}$ \\
\hline
\end{tabular}

\begin{tabular}{|ll|}
\hline - & Dificultad en la memoria declarativa \\
semántica \\
- Dificultad en el reconocimiento y \\
procesamiento de emociones \\
- Dificultad en el procesamiento verbal \\
- Dificultad en la memoria espacial y \\
- episódica \\
- Demoria de trabajo verbal limitada \\
visuoespacial \\
\hline Características conductuales \\
- Dificultad en la socialización \\
- dimitaciones en habilidades de la vida \\
- Comunicación interpersonal deficiente \\
Dificultad en la adaptabilidad en el contexto \\
\hline
\end{tabular}

\section{Cognición social y trisomía 21}

Finalmente, existen observaciones clínicas en pacientes con trisomía 21, que dan pauta a pensar que no solo tienen una interacción social intacta, sino que su interacción con desconocidos se da de una forma natural. Esto ha llevado a pensar que su interacción social atípica podría estar relacionada con un distinto desarrollo de la Cognición Social (CS). La CS es el conjunto de procesos neuropsicológicos que se ven comprometidos en situaciones donde involucra un contexto y la interacción social. Una de las áreas más estudiadas de la CS es la teoría de la mente (ToM). Premack y Woodruff (1978) propusieron el término teoría de la mente que hace referencia a la habilidad para describir, asignar, atribuir estados mentales a otros y a uno mismo, con la evolución de las neurociencias se ha encontrado que la parte prefrontal es la responsable de la función ejecutiva de la cognición social (Rains, 2004), inicialmente surge el constructo de teoría de la mente para explicar el Trastorno del Espectro Autista (TEA), sin embargo se ha observado que pueden existir alteraciones clínicas derivadas de las funciones alteradas de los lóbulos frontales, de acuerdo con Sabbagh y Bowman (2018) el correcto desarrollo de la teoría de la mente en humanos es indispensable ya que cualquier alteración se va a manifestar en severas dificultades de la interacción social, dentro de un contexto en específico, básicamente la capacidad de poder interpretar el estado anímico del otro de forma automática e inmediata nos permite solucionar problemas, interactuar de forma positiva y conseguir nuestros propios intereses. De acuerdo con las investigaciones de Adholps (2001:2005) a continuación se describirán brevemente algunas de las regiones cerebrales involucradas en la ToM:

- Amígdala: participa en el procesamiento de emociones básicas (se activa ante variables que 
operan en el contexto) y de emociones sociales (por ejemplo, en relaciones interpersonales).

- Cuerpo estriado ventral: se activa con aspectos motivacionales cuando se percibe atractivo un rostro o la cara de una persona y se relaciona con las funciones ejecutivas frías del comportamiento moral.

- Giro fusiforme y giro temporal superior: procesa propiedades estructurales y estáticas de las caras. En conjunto con la amígdala y el hipocampo, se activa ante la presencia de rostros que expresan miedo, lo que sugiere un circuito de procesamiento de información en la que estos últimos recuperan memorias episódicas guardadas en el área fusiforme de rostros.

- El giro temporal superior se encuentra involucrado en el procesamiento de la expresión facial, la percepción de la voz, y se relaciona con los déficits atribucionales.

- Corteza somatosensorial: en esta área se realiza la representación perceptual del estímulo, lo que permite tener un panorama de lo que sucede en el cuerpo, para enviar información a las funciones ejecutivas y así tomar decisiones conscientes.

- Polo temporal: se encarga de la elaboración de los juicios morales simples, introspección de eventos autobiográficos con contenido emocional y, en junto con la ínsula y el precuneus, integran un circuito de atribución emocional.

- Circunvolución del cíngulo: proporciona información necesaria para que se realice el control consciente de la emoción y el comportamiento moral a través de la recuperación de información y la imaginación de situaciones emocionales.

- Ínsula: interpreta signos emocionales evidentes, lo que facilita la empatía.

- Corteza prefrontal media: junto con la corteza orbitrofrontal, se encarga de regular la conducta en casos de cooperación social, comportamiento moral y agresión social (se activa con la transgresión a la norma).

- Corteza prefrontral orbitral: se encarga de la comprensión de los sentimientos de otras personas y, por lo tanto, el eje de la empatía, realizar la representación emocional de las metas.

- Corteza prefrontal dorsomedial: se realiza la codificación de información social.

Téllez-Vargas (2006) propone un esquema (Esquema 1) de las principales estructuras cerebrales que conforman la teoría de la mente y su relación.

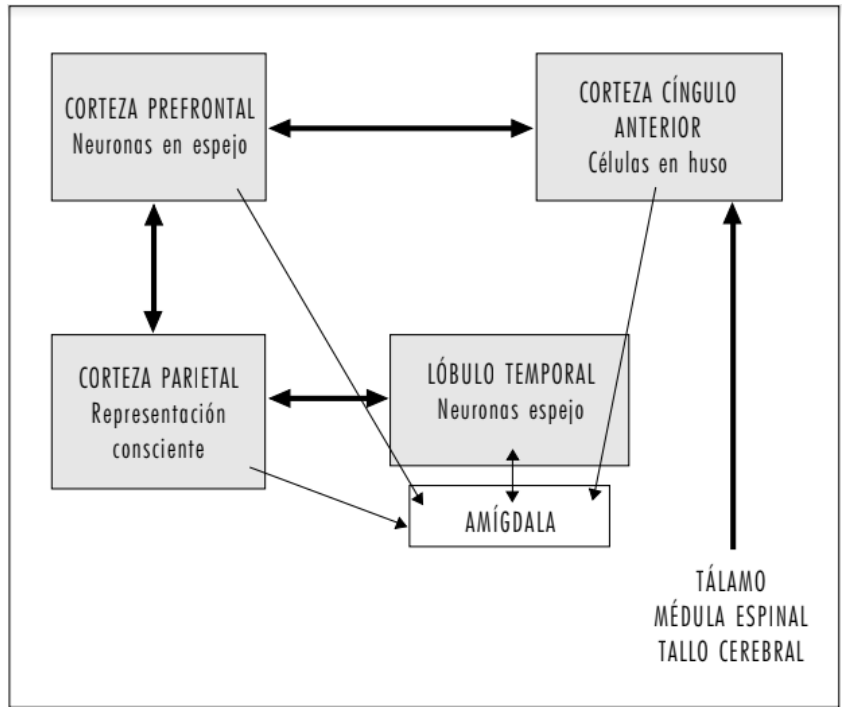

Esquema 1. Principales estructuras cerebrales involucradas en el sustrato neurobiológico para la teoría de la mente

Las funciones neurobiológicas descritas a nivel cortical y subcortical han dado pauta para indagar cuales son las funciones cognitivas de la CS que se podrían ver afectadas en niños con trisomía 21, cabe mencionar, que el estudio de la CS es incipiente en este tipo de población además de que aún no se hallan instrumentos estandarizados que permitan la evaluación de la CS en trisomía 21.

A continuación, se describirán algunos hallazgos que se ha encontrado en trisomía 21.

- Yirmiya y Shulman en 1996; Baron-Cohen en 1989 (citado en Górriz, 2004) describieron que los niños con SD aparentemente no tienen dificultad con las tareas de teoría de la mente, sin embargo, al verse comparados con niños neurotípicos la ejecución no es la ideal como los niños con desarrollo típico, esto podría explicarse por las limitaciones en el procesamiento de información.

- Estudios como los de Baron-Cohen, Leslie y Frith (1985) encontraron que los niños con SD no presentan dificultad en la tarea de falsa creencia, además reflejan mayor competencia social en comparación a niños con autismo, identifican los sentimientos de los demás, son capaces de entender las intenciones y de comprender las mentiras, con un poco de lentitud en el procesamiento de toda la información, sin embargo en el contenido emocional no distinguen una emoción positiva de una negativa en sí mismos. 
- Górriz (2004) menciona que los niños con SD presentan dificultad en la comprensión de peticiones e insinuaciones, esto se explicaría porque el lenguaje expresivo está relacionado con los estados mentales, por lo que en niños con SD con dificultad en el lenguaje expresivo se manifiesta por su poca capacidad mentalista. Sin embargo, acorde con el autor diversas investigaciones señalan que la teoría de la mente en sujetos con SD sigue siendo bastante débil en comparación a las investigaciones en Trastorno del Espectro Autista.

- Binnie y Williams (2010) estudiaron niños prescolares típicos, con TEA y SD y los evaluaron en las tareas de creencia falsa de Baron-Cohen, donde describen que los niños con SD tienen una respuesta pobre en este tipo de tareas.

- Cebula, Moore y Wishart (2010) realizan un análisis teórico de la construcción de la cognición social en niños con SD, donde mencionan la importancia de estudios longitudinales que puedan explicar las variables sociocognitivas en la discapacidad intelectual y que exista la adaptación de instrumentos de manera particular a niños con SD para comprender su CS.

- Martinez-Castilla et al. (2014) estudiaron el reconocimiento de emociones faciales en individuos con Síndrome de Williams (SW), SD y desarrollo típico (DT), donde encontraron que hay mayor facilidad de reconocer emociones positivas en SW y SD, y que el reconocimiento de emociones faciales negativas se atrasa en $S D$, pero sin ser significativo en comparación con SW y DT.

- Amado, Serrat y Vallés-Mejoral (2016) evaluaron CS en niños con SD y DT, encontraron un desempeño inferior en comparación a sus compañeros con un DT, tanto en términos de CS como de Funciones Ejecutivas (FE) principalmente resaltando el papel de la memoria de trabajo.

Pochon, Touchet y Ibernon (2017) evaluaron el reconocimiento facial de emociones en adolescentes con SD y con DT, sin hacer uso del vocabulario emocional, el análisis de sus resultados, no encontraron diferencias significativas en el reconocimiento de expresiones faciales con emociones.

\section{Referencias}

[1] Adolphs, R. (2001). The neurobiology of social cognition. . Curr Opin Neurobiol.

[2] Adolphs, R., Gosselin, F., Buchanan, T., Trenel, D., Schyns, P., \& Damasio, A. (2015). A mechanism for impaired fear recognition after amygdala damage. Nature.

[3] Altafaj, X., Dierssenm, M., Baamonde, C., Martí, E. y Visa, J., (2001). Neurodevelopmental delay, motor abnormalities and cognitive deficits in transgenic mice overexpressing Dyrk1A (minibrain), a murine model of Down's syndrome. Human Molecular Genetics, Volume 10, Issue 18, 1 September 2001, Pages 1915-1923, https://doi.org/10.1093/hmg/10.18.1915

[4] Amadó, A., Serrat, E., and Vallès-Majoral. (2016). The Role of Executive Functions in Social Cognition among Children with Down Syndrome: Relationship Patterns. 19 Diciembre 2018, de Frontiers In Psychology Sitio web: https://doi.org/10.3389/fpsyg.2016.01363

[5] Asociación Psiquiatría Americana (2014). Manual estadístico de los trastornos mentales. 5ta Ed., México; Panamericano..

[6] Baron-cohen, S., Leslie, A., y Frith, U. (1985) does the autistic child have a "theory of mind"? Cognition, 21, 37-46

[7] Boletín Médico del Hospital Infantil (2014). Prevalencia del síndrome de Down en México utilizando los certificados de nacimiento vivo y de muerte fetal durante el periodo 2008-2011. [consultado 16 agosto 2018]. Disponible en: http://www.scielo.org.mx/pdf/bmim/v71n5/v71n5a6.pdf

[8] Cebula K., Moore D., y Wishart, J. (2010). Social cognition in children with Down's syndrome: challenges to research and theory building. 17 Diciembre 2018, de Journal of Intellectual Disability Research Sitio web: https://doi.org/10.1111/j.13652788.2009.01215.x

[9] Gibbs, M. y Thorpe, J. (1983). Personality stereotype of noninstitutionalized Down syndromechildren. American Journal of Mental Deficiency, 601-605.

[10] Godoy Briceño, J. y Campos Pardo, F. (2014). Descripción del nivel de desarrollo psicomotor en niños con síndrome de Down: creación de una tabla descriptiva. [consultado 16 agosto 2018]. Disponible http://www.down21materialdidactico.org/PDFDown21/chileD SM.pdf

[11] Goldman, K., Shulman, Y., Bar-Haim, R., Abend, And J.A. Burack. (2016). Attention allocation to facial expressions of emotion amongpersons with Williams and Down syndromes. 16 Diciembre 2018, de Development and Psychopathology Sitio web: http://10.1017/S0954579416001231

[12] Górriz Plumed (2004). Jornades de foment de la investigació; teoría de la mente y síndrome de Down: Revisión teórica. Universitat Jaume. Disponible en: http://ardilladigital.com/DOCUMENTOS/DISCAPACIDADE S/SINDROME\%20DE\%20DOWN/EDUCACION\%20Y\%20P SICOLOGIA/PSICOLOGIA/Teoria $\% 20 \mathrm{de} \% 201 \mathrm{a} \% 20$ mente $\% 2$ 0y\%20SdD\%20-\%20Jaime\%20I\%20-\%20articulo.pdf

[13] Grego Del Col, C., Cavalcante Caetano, S., Ribeiro W. y Kummer, A. (2017). Adolescent adaptive behavior profiles in Williams-Beuren syndrome, Down syndrome, and autism spectrum disorder. 18 Diciembre 2018, de Child Adolescent Psychiatry and Mental Health Sitio web: https://doi10.1186/s13034-017-0177-0

[14] Jack S. Krasuski , M.D., Gene E. Alexander , Ph.D., Barry Horwitz , Ph.D., Stanley I. Rapoport , M.D., and Mark B. Schapiro , M.D.. (2002). Relation of Medial Temporal Lobe Volumes to Age and Memory Function in Nondemented Adults With Down's Syndrome: Implications for the Prodromal Phase of Alzheimer's Disease. 17 Diciembre 2018, de The American Journal of Psychiatry Sitio web: https://doi.org/10.1176/appi.ajp.159.1.74

[15] Lynne M. Binnie Joanne M. Williams. (2010). Intuitive psychological, physical and biological knowledge in typically developing preschoolers, children with autism and children with Down's syndrome. 18 Diciembre 2018, de British Journal of Developmental Psychology Sitio web: https://doi.org/10.1348/026151002320620361

[16] Määttä T, Tervo-Määttä T, Taanila A, Kaski M, Iivanainen M. (2006). Mental health, behaviour and intellectual abilities of 
people with Down syndrome. 17 diciembre 2018, de PublMed.gov Sitio web: https://www.ncbi.nlm.nih.gov/pubmed/17048808

[17] Martínez-Castilla, P., Burt, M., Borgatti \& Chiara Gagliardi. (2014). Facial emotion recognition in Williams syndrome and Down syndrome: A matching and developmental study. 18 Diciembre 2018, de Journal Child Neuropsychology Sitio web: https://doi.org/10.1080/09297049.2014.94540

[18] Michael S. Schimmel, Cathy Hammerman, Ruben Bromiker, Itai Berger. (2006). Third Ventricle Enlargement Among Newborn Infants With Trisomy 21. 17 Diciembre 2018, de AAP New \& Journals Sitio web: http://pediatrics.aappublications.org/content/117/5/e928?sso=1 \&sso_redirect_count $=3 \&$ nfstatus $=401 \&$ nftoken $=00000000$ 0000-0000-0000-

$000000000000 \&$ nfstatusdescription=ERROR $\% 3 \mathrm{~A} \% 20 \mathrm{No} \% 20$ ocal\%20token\&nfstatus $=401 \&$ nftoken $=00000000-0000-0000$ $0000-$

000000000000\&nfstatusdescription $=$ ERROR $\% 3 a+N o+l o c a l+t$ oken

[19] Morales, G., \& López, E. (2006). El síndrome de down y su mundo emocional. México: Trillas.

[20] National Institute of Child Health and Human Development (2018). Down syndrome. [consultado 16 agosto 2018]. Disponible https://www.nichd.nih.gov/health/topics/downsyndrome

[21] Organización Mundial de la Salud (2014). Día mundial del síndrome de Down, 21 de marzo. Antecedentes [consultado 16 agosto 2018]. Disponible en http://www.un.org/es/events/downsyndromeday/background.sh tml

[22] Perry, J. C., Pakkenberg, B., \& Vann, S. D. (2018). Striking reduction in neurons and glial cells in anterior thalamic nuclei of older patients with Down's syndrome. Neurobiology of Aging.

[23] Pinter, J. D., Brown W. E., Eliez, S., Schmitt, G. T., Capone, A L. Reiss. (2001). Amygdala and hippocampal volumes in children with Down syndrome: A high-resolution MRI study. 17 Diciembre 2018, de ANN Sitio web: https://doi.org/10.1212/WNL.56.7.972

[24] Pochon, R., Touchet, C., \& Ibernon, L. (2017). Emotion Recognition in Adolescents with Down Syndrome: A Nonverbal Approach. Brain Sci.

[25] Premack D. y Woodruff, G. (1978). Does chimpanzee have theory of mind? Behav Brain Sci 1978; 4: 9-30

[26] Pueschel, S. y Pueschel, J. (1994). Síndrome de Down. Problemática biomédica. Barcelona, España. Editorial Masson S.A. 1ra Edición.

[27] Rains, D. (2004). Principios de neuropsicología humana. 1ra Edición. México: Mc Graw Hill.

[28] Raz, N., Torres, I. J., Briggs, S. D., Spencer W. D., Thornton A E., Loken W. J., Gunning M, F., McQuain J. D., Driesen, Acker, J. (1995). Selective neuroanatornic abnormalities in Down's syndrome and their cognitive correlates Evidence from MRI morphometry. 17 Diciembre 2018, de AAN Sitio web: https://doi.org/10.1212/WNL.45.2.356

[29] Sabbagh, M. A., \& Bowman, L. C. (2018). Thory of Mind Developmental and Social Psychology.

[30] Secretaría de Salud de México. Centro Nacional de Equidad de Género y Salud Reproductiva. Atención integral de la persona con síndrome de Down. Lineamiento técnico. Secretaría de Salud 2007 [consultado 16 agosto 2018]. Disponible en: http://www.salud.gob.mx/unidades/cdi/documentos/Síndrome Down lin 2007.Pdf

[31] Silvia Lanfranchi, Cesare Cornoldi, Renzo Vianello. (2004). Verbal and Visuospatial Working Memory Deficits in Children
With Down Syndrome. 18 Diciembre 2018, de American Association on Intellectual and Developmental Disabilities Sitio web: http://www.aaiddjournals.org/doi/abs/10.1352/08958017(2004)109\%3C456:VAVWMD\%3E2.0.CO;2

[32] Snell, S. (2001). Neuroanatomía clínica. 5ta Ed. México: Panamericano

[33] Strafford, B. (1998). Síndrome de Down: pasado, presente y futuro. $1^{\text {a }}$ edición. México; edivisión.

[34] Téllez-Vargas, J. (2006). Teoría de la mente: evolución, ontogenia, neurobiología y psicopatología. Avances de psiquiatria Biológica.

[35] Wu, J., Morris, J, K., (2013). La prevalencia de la población del síndrome de Down en Inglaterra y Gales en 2011. EUR: Gineta. 